\title{
Performance Analysis of Multi Traffic over Multi Rate EDCA of WLAN Network
}

\author{
Meenal $^{1}$ and Kiran Ahuja ${ }^{2}$ \\ ${ }^{1,2}$ Electronic and Communication, DAVIET \\ Punjab, India \\ Imeenal2707@gmail.com,2askahuja2002@gmail.com
}

\begin{abstract}
Enhanced distributed channel access (EDCA) is used for transmitting good quality of service of multimedia traffic in wireless local area network (WLAN). Applications like Constant bit rate (CBR), file transfer protocol (FTP) and voice over internet protocol (VoIP) are used in wireless LAN network. Audio data is assign as high priority (AC_VO) and other such as CBR \& FTP type of data are assigned as low priority (AC_BE) in IEEE 802.11b network. G.711 provides a very good perceived audio quality in comparison to other audio CODECs. The G711 codec is used in VoIP call with 20 msec of audio data. In this paper, analysis of the effects on CBR\& FTP traffic with increased call rate of VOIP and payload sizes of CBR \& FTP traffic in WLAN environment for real time applications are made. Jitter buffer used in RTP protocol is improving the quality of service (QoS) of multi traffic in comparison to RTP protocol without jitter buffer.
\end{abstract}

Keywords: EDCA, G.711 Codec, VoIP, CBR, FTP, priority, RTP protocol, jitter buffer

\section{Introduction}

Wireless LAN [1] technology provides a very good business model as it uses free unlicensed frequencies and provides a wireless last hop to IP networking which is also free. The IEEE 802.11 standard provides two MAC methods: Distributed Coordination Function (DCF) and Point Coordination Function (PCF). The original standard IEEE 802.11 failed to provide the required quality of service $(\mathrm{QoS})$ performance as it serves all transmitted frames with the same level of priority. To provide a better QoS a new standard called IEEE 802.11e was deployed by enhancing the original standard [2].

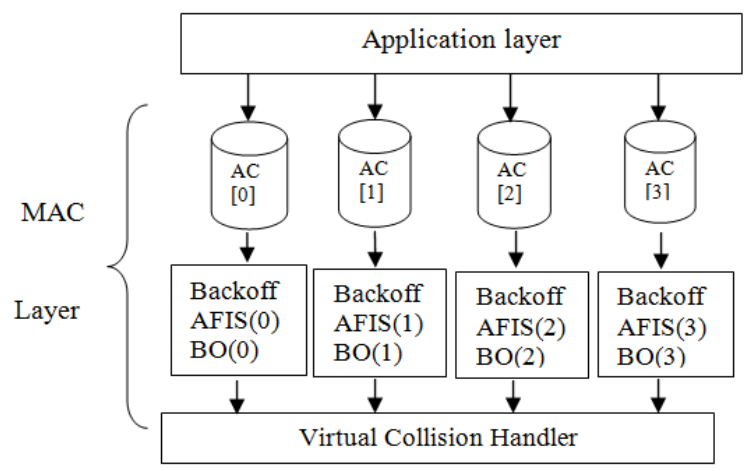

Figure 1. IEEE 802.11 Architecture 
Table 1. 802.11e EDCA Parameters

\begin{tabular}{|c|c|c|c|c|}
\hline Parameter & AC [0] =BK & AC [1] = BE & AC [2]= VI & AC[3] =VO \\
\hline Precedence & 0 & 1,2 & $3,4,5$ & 6,7 \\
\hline AIFS & 7 & 3 & 2 & 2 \\
\hline TXOPlimit & 0 & 0 & $6.016 \mathrm{~ms}$ & $3.264 \mathrm{~ms}$ \\
\hline Cwmin & 31 & 31 & 15 & 7 \\
\hline Cwmax & 1023 & 1023 & 31 & 15 \\
\hline
\end{tabular}

In multi-rate operation, Access point (AP) selects the appropriate transmission rate from a set of possible rates based on wireless channel conditions. Algorithms for multi-rate operation are broadly classified into two categories [3]: Signal-to-Noise-Ratio (SNR) measurement based and Statistical Count based. SNR measurement based schemes include Automatic Rate Fallback (ARF) algorithm which measures SNR at the receiver and convey it to the transmitter. ARF algorithm is implementing with channel parameters as shown in Table 2.

Table 2. 802.11b Physical Layer Parameters for Multi-Rate

\begin{tabular}{|c|c|}
\hline data rate & Receiver sensitivity \\
\hline $1 \mathrm{Mbps}$ & $-94.9 \mathrm{dbm}$ \\
\hline $2 \mathrm{Mbps}$ & $-91.0 \mathrm{dbm}$ \\
\hline $5.5 \mathrm{Mbps}$ & $-87.0 \mathrm{dbm}$ \\
\hline $11 \mathrm{Mbps}$ & $-83.0 \mathrm{dbm}$ \\
\hline
\end{tabular}

The paper is organized as follows. Section 2 represents related work. Section 3 defines proposed scenario. Section 4 defines performance evaluation parameters. Section 5 shows results and discussion. Section 6 includes conclusion and future work.

\section{Related Work}

The performance of the IEEE $802.11 \mathrm{~b}$ wireless local area networks has been analyzed in previous research work. The performance of IEEE 802.11e EDCA is very effective service as compared with IEEE 802.11 DCF From the results, it was concluded that IEEE 802.11e introduces a very effective service differentiation mechanism to provide QoS support. Evaluated at heavy load of data has been high priority ACs i.e., AC_VO and AC_VI suffer from greater number of collisions due to small CWmin and CWmax values [4]. Different traffic types like CBR, VBR and FTP are used to completely analyze the performance differences between two standards (DCF and EDCA). The enhanced standard (EDCA) is found better in protecting and providing less delay in medium access to high priority flows [5]. In case of EDCF, as there was a separate queue for each priority traffic, a minor change in throughput, latency and drop ratio was observed for voice as compared to DCF [6].The EDCA QoS mechanism produced significant improvements in the transmission of voice, which was consequential in the case of highly loaded network. Measurement results had shown that the use of QoS mechanisms reduced values of the mean and maximum delay, jitter and lost packets as compared to the case without QoS mechanism [7].When G711 VoIP connections is reduction in the bandwidth available for data traffic by approximately 900 Kbps [8].The MOS (mean opinion score) value of different VOIP CODECs (G.711) found was 4.48322 without background traffic (data) over 802.11e and 802.11b [9] Dynamic jitter buffer schemes give the highest user satisfaction in VoIP codec (G.711) and WLAN environments where compared without dynamic jitter buffer schemes [10].

The current work is distinguished from a majority of the previous literature. First, the performance of the CBR and FTP generic traffic by changing the parameters CBR and FTP 
generic traffic respectively. Finally, we measurement the performance of CBR \& FTP generic traffics by increasing connection of VoIP with and without jitter buffer is done. Therefore the research work represents performance of different types of traffics by using or not jitter buffer in VoIP traffic.

\section{Proposed Scenario}

Simulations have been performed in QualNet 5.2 to deploy multi traffic application in a single QoS-enabled Basic Service Set (QBSS). Multi traffic such as constant bit rate (CBR), file transfer protocol (FTP) and voice over internet (VoIP) traffic studies are done in this paper. Eight stationary nodes (stations) are considered with single Access point, a single channel and single basic service set (BSS), which is a reasonable assumption of capacity. All even stationary nodes are assumed as sender and all odd nodes are assumed as destination whereas one node is assumed as AP. We have developed a system model for evaluating the performance of multi traffic over multi rate EDCA of WLAN.

A generic term used to describe the techniques used to carry voice traffic over IP. G.711 codec used in VoIP. In order to establish H.323 connections, two protocols RTP and RTCP must be set up between the two devices. RTP/RTCP protocols are defined in RFC 3550 [11].

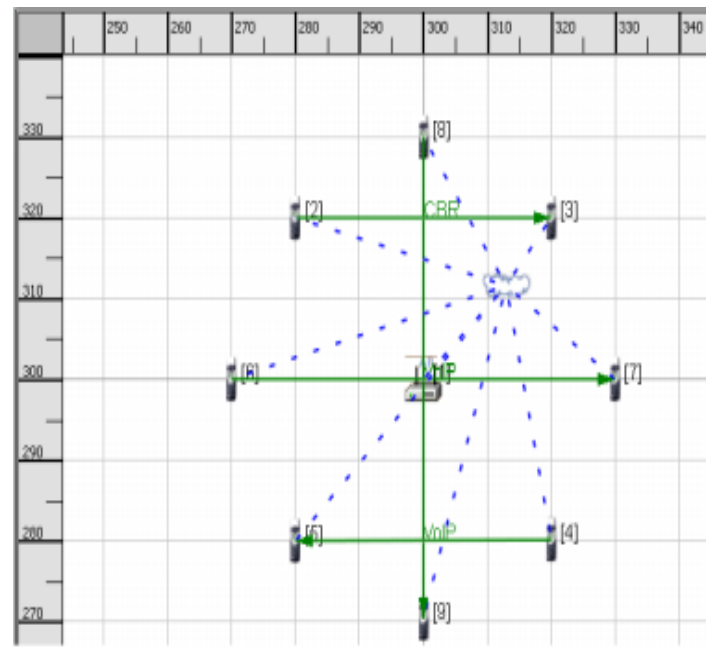

Figure 2. Network Topology for Stationary Nodes with Multi Traffics

Single CBR or FTP traffic transmits packets from node 2 (server) to node 3 (client) with different parameters of CBR or FTP traffic respectively. Analysis of the parameter of QoS of CBR \& FTP is made. Evaluation of the performance of a single traffic with increasing call rate over multi rate EDCA of WLAN is done. Total three calls are established in it. First call is established between Node 6 (client) to node 7 (server). Second call is established between Node 4 (client) to node 5 (server).Third call is established between node 8 (client) to node 9 (server). A single CBR traffic transmits 1000 packets with 1472 bytes in 5 msec. A single FTP traffic transmits 1000 packets with 1472 bytes. Time for simulation is 6 minutes. 
Table 3. Parameters of Multi Traffic

\begin{tabular}{|c|c|c|c|}
\hline $\begin{array}{c}\text { Type of } \\
\text { traffic }\end{array}$ & \multicolumn{3}{|c|}{ Parameter value } \\
\hline & Payload size & Number of packet & Time interval \\
\hline CBR & 1472 bytes & 1000 packets & $5 \mathrm{~ms}$ \\
\hline FTP/GEN & 1472 bytes & 1000 packets & - \\
\hline VoIP & G.711 CODEC (160 bytes per packet at $20 \mathrm{~ms})$ \\
\hline
\end{tabular}

Table 4. Simulation Parameters

\begin{tabular}{|c|c|}
\hline Parameters & Value \\
\hline Terrain Size & $600 * 600$ meters \\
\hline Physical protocol & IEEE $802.11 \mathrm{~b}$ \\
\hline MAC protocol & IEEE 802.11e,EDCA enable \\
\hline Short Packet Transmit Limit & 7 \\
\hline Long Packet Transmit Limit & 4 \\
\hline MAC Propagation Delay & $1 \mu \mathrm{s}$ \\
\hline Beacon Interval & $200 \mathrm{TU}$ \\
\hline Network Protocol, IPV4 & Fragment Unit 2048 bytes, Hold time $15 \mathrm{sec}$ \\
\hline Number of IP Output Queues & 3 with strict priority \\
\hline Routing Protocol & Bellman Ford \\
\hline IP Input Queue Size & 150 Kbytes \\
\hline Transport Protocol & RSVP, UDP and TCP-lite enabled \\
\hline Maximum Segment Size & 512 Byte \\
\hline Send/Receive Buffer Size & 16384 bytes buffer \\
\hline QoS application & VoIP, CBR \& FTP generic \\
\hline Average time & $20 \mathrm{sec}$ \\
\hline Connection Delay & $8 \mathrm{sec}$ \\
\hline Call Timeout & $60 \mathrm{sec}$ \\
\hline Packetization Interval & $20 \mathrm{msec}$ \\
\hline Total Loss Probability & 5.07 \\
\hline Multimedia signaling & H323: Direct call model, No Gatekeeper \\
\hline RTP & Enabled \\
\hline RTCP Session Bandwidth & 64 Bps \\
\hline
\end{tabular}

\section{Performances Evaluation}

The following QoS performance metrics of multi traffic are considered for performance evaluation [11].

4.1. Throughput: It is the number of bits passed through a network in one second. The throughput of the network is finally defined as the average of the throughput of all nodes involved in data transmission. Therefore, the throughput can be stated as:

$$
\text { Throughput }=\frac{(\text { total bytes received } * 8)}{(\text { session ended at }- \text { session initiated at })}
$$


4.2. Average End-to-End Delay: Average End-to-End delay indicates the length of time taken for a packet to travel from source to the destination. The average delay is calculated by taking the average of delays for every data packet transmitted.

$$
\begin{aligned}
& \text { Avg. delay }_{\text {end-end }}=\frac{(\text { Total trans. delay of all received pkts })}{(\text { Number of pkts received })} \\
& \text { Trans. delay }=\left(\text { Time }_{\text {pkts received at server }}-\text { Time }_{\text {pkts transmitted at client }}\right)
\end{aligned}
$$

4.3. Average Jitter: Jitter is the difference of packet transit delays between two consecutive packets. Thus early, late, or out of sequence arrival of packets will cause jitter.

$$
\text { Avg. } \text { Jitter }_{\text {end }- \text { end }}=\frac{(\text { Total pkt jitter of all received pkts })}{(\text { Number of pkts received }-1)}
$$

4.4. Packet Loss Rate: The difference of total packets sent and total packets received give the total packet loss. Packet loss is related to delivery ratio as follows:

$$
\begin{gathered}
\text { PDR }(\%)=\frac{(\text { Total pkt received })}{(\text { Total pkts sent })} \times 100 \\
\operatorname{PLR}(\%)=100-\text { packets Delivery Ratio }(\text { PDR })
\end{gathered}
$$

\section{Results and Discussion}

This section include following results of multi traffic with different parameters of multi traffic over EDCA.

\subsection{Increase Payload Size of Traffic}

The simulation results indicate that the maximal achievable payload data rate of CBR and FTP traffic in this scenario is approximately $2.4 \mathrm{Mbps}$ and $0.741 \mathrm{Mbps}$ respectively which is achieved when the payload size of traffic is chosen to be 1472 bytes. With an increase the payload size in each packet of traffic, the number of bits in each packet also increases. As a result, increasing the packet size of traffic will increase the throughput of traffic whereas average delay and average jitter of traffic decreases.

As show in the graph payload size of each packet of CBR \& FTP traffic is varies 50 bytes to 1500 bytes. Figures 4, 5 and 6 shows the result of different parameters of QoS of multi traffic (CBR \& FTP) when payload size of each packet is increased in a single sender node over multi rate EDCA and 802.11b.

5.1.1. Effect on Throughput of Multi Traffic: The throughput of CBR traffic increases and varies from $80.8 \mathrm{kbps}$ to $2.40 \mathrm{Mbps}$ and also FTP traffic varies from $0.83 \mathrm{Mbps}$ to 0.741 Mbps. 


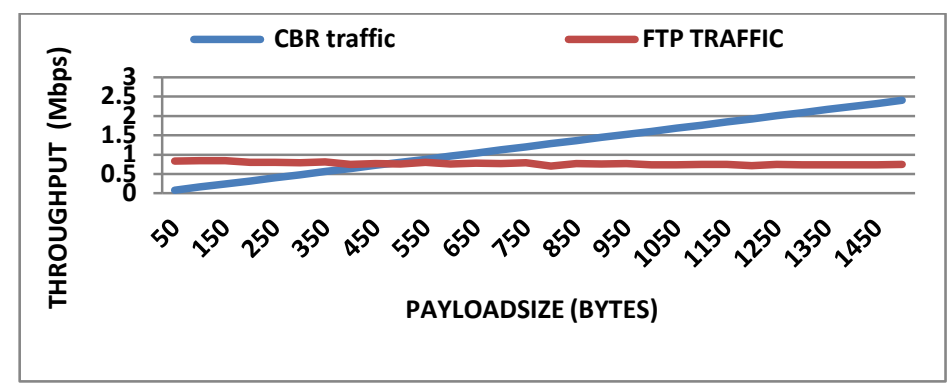

Figure 4. Throughput Vs Payload Size of Traffic

5.1.2. Effect on Average Delay of Multi Traffic: Average delay of CBR traffic varies 2.61 msec to $11.66 \mathrm{msec}$ and also FTP traffic varies from $119.23 \mathrm{msec}$ to $98.73 \mathrm{msec}$.

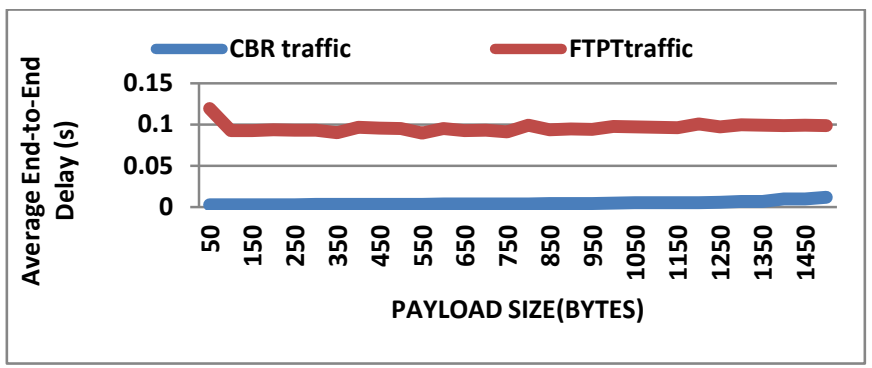

Figure 5. Average End to End Delay Vs Payload Size of Traffic

5.1.3. Effect on Average Jitter of Multi Traffic: Average jitter of CBR traffic varies from $0.313 \mathrm{msec}$ to $2.22 \mathrm{msec}$ and also FTP traffic varies from $0.827 \mathrm{msec}$ to $8.20 \mathrm{msec}$.

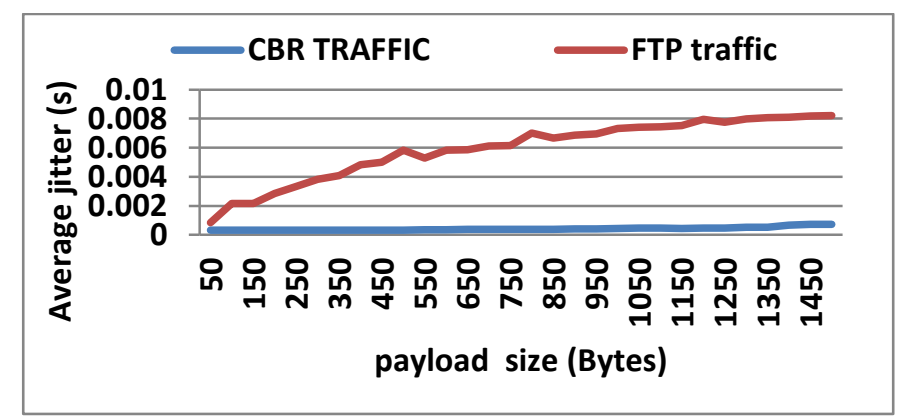

Figure 6. Average Jitter Vs Payload Size of Traffic

\subsection{Increased Calls Rate}

Firstly three VoIP calls are established without using background data such as CBR \& FTP traffic in WLAN network. G711 codec used in VoIP, each frame is transmitted from source to destination within 20ms. IP/UDP/RTP protocols are used to send the codec source to destination. The audio data packets are sent every $20 \mathrm{~ms}$ and payload of each packet is 160 bytes. Analysis show that maximum throughput of G 711 codec is $39.293 \mathrm{Kbps}$, Mean opinion score (MOS) of G711 is 3.29 and Packet loss is $2 \%$ when three calls are established. For the three calls, the quality of all connections is good. Thus, the experiment indicates number of VoIP connections is 3 in a single cell of an IEEE 802.11b network. 
Multi traffic such as voice and data traffic are considered in the wireless network. One station transmits the data traffic such as CBR \& FTP, whereas other stations transmit voice data from sender to destination. All connection terminals are established in the wireless network. Table 3 shows observation of different performance of CBR and FTP traffic with increasing VoIP call rate. Figures 7, 8, 9 and 10 shows the result of different parameter of QoS of multi traffic when increase call rate using with and without jitter buffer.

5.2.1. Effect on Throughput of Multi Traffic: Throughput of CBR \& FTP traffic decreases and average delay and average jitter increased with increase call rate. Throughput of CBR and FTP traffic is $2.36 \mathrm{Mbps}$ and $0.679 \mathrm{Mbps}$ respectively without call established. Throughput of CBR traffic is $2.36 \mathrm{Mbps}, 2.06 \mathrm{Mbps}, 1.977 \mathrm{Mbps}$ and $1.624 \mathrm{Mbps}$ without using jitter buffer in RTP protocol and 2.36 Mbps, 2.06 Mbps, 2.04 Mbps and 1.719 Mbps with using jitter buffer in RTP protocol when increase call rate $(0,1,2$, and 3$)$ in this scenario.

Throughput of FTP traffic is $0.679 \mathrm{Mbps}, 0.635 \mathrm{Mbps}, 0.563 \mathrm{Mbps}$ and $0.442 \mathrm{Mbps}$ without considering jitter buffer and $0.679 \mathrm{Mbps}, 0.635 \mathrm{Mbps}, 0.566 \mathrm{Mbps}$ and $0.456 \mathrm{Mbps}$ with jitter buffer when increase call rate $(0,1,2$, and 3$)$. All observation shows in Figure 7 . It is analyzed that with an increased call rate, the number of packets in VoIP traffic also increases. It affects the throughput of traffics such as CBR \& FTP.

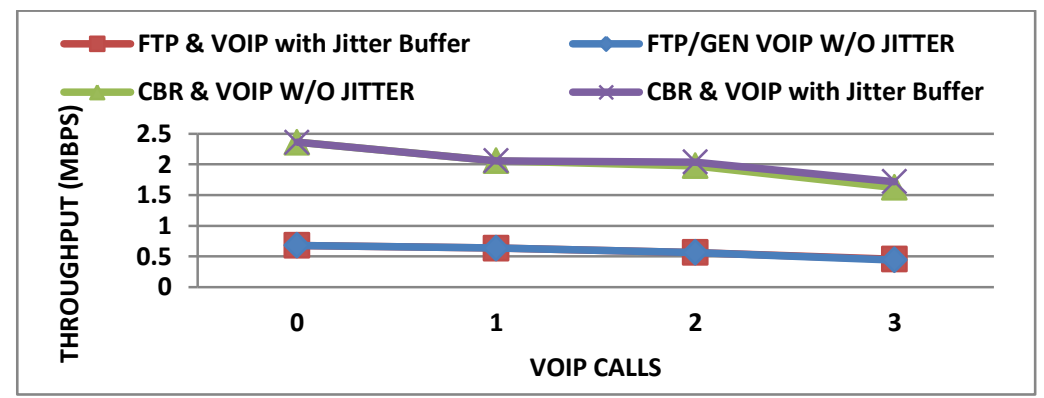

Figure 7. Throughput Vs VolP Calls

5.2.2. Effect on Average Delay of Multi Traffic: Average delay of CBR and FTP traffic varies with increased call rate. Average delay of CBR traffic is $0.038598 \mathrm{~s}$ without call establishment. Average delay of CBR traffic is $0.038598 \mathrm{~s}, 0.399503 \mathrm{~s}, 0.532191 \mathrm{~s}$ and $0.562358 \mathrm{~s}$ without consider jitter buffer in RTP protocol and $0.038598 \mathrm{~s}, 0.399503 \mathrm{~s}, 0.4974 \mathrm{~s}$ and $0.537055 \mathrm{~s}$ with consider jitter buffer in RTP protocol when increased call rate.

Average delay of FTP traffic is $0.008889 \mathrm{~s}, 0.009586 \mathrm{~s}, 0.010801 \mathrm{~s}$ and $0.013773 \mathrm{~s}$ with increase call rate without jitter buffer and $0.008889 \mathrm{~s}, 0.009586 \mathrm{~s}, 0.010743 \mathrm{~s}$ and $0.013348 \mathrm{~s}$ with increase call rate with jitter buffer. Average delay of CBR traffic is more increase as compare FTP traffic. Average delay is increasing with decrease throughput.

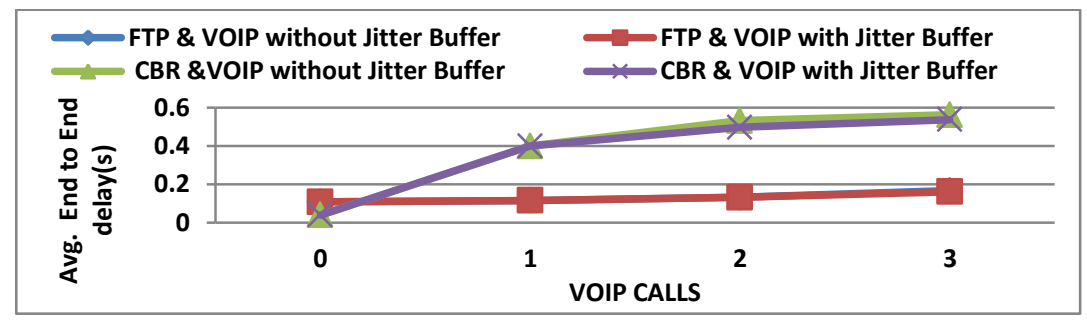

Figure 8. Average End to End Delay Vs VolP Calls 
5.2.3 Effect on Average Jitter of Multi Traffic: Average jitter of CBR and FTP traffic also changes with increased call rate. Average jitter of CBR and FTP traffic is 0.001091s and $0.107346 \mathrm{~s}$ respectively without any call establishment. Average jitter of CBR traffic is $0.001091 \mathrm{~s}, 0.00276 \mathrm{~s}, 0.003064 \mathrm{~s}$ and $0.004124 \mathrm{~s}$ with increase call rate $(0,1,2$, and 3$)$ without jitter buffer and $0.001091 \mathrm{~s}, 0.00276 \mathrm{~s}, 0.002684 \mathrm{~s}$ and $0.003887 \mathrm{~s}$ with increase call rate with jitter.

Average jitter of FTP traffic is $0.107346 \mathrm{~s}, 0.114899 \mathrm{~s}, 0.131762 \mathrm{~s}$ and $0.166481 \mathrm{~s}$ with increase call rate without jitter buffer and 0.107346s, 0.114899s, $0.131416 \mathrm{~s}$ and $0.160754 \mathrm{~s}$ with increased call rate with jitter buffer. Average jitter of CBR and FTP traffics are decreasing with increasing throughput of CBR and FTP traffic.

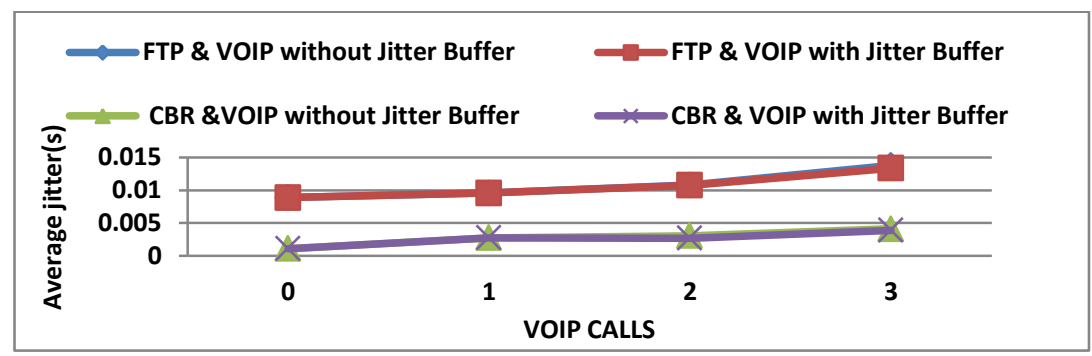

Figure 9. Average Jitter Vs VolP Calls

5.2.4. Effect on Packet Loss Rate of Multi Traffic: Variation of packet loss rate of CBR is also observed with increase call rate. No packet loss rate in CBR and FTP traffic without any calls is observed. Packet loss rate of CBR traffic is $0 \%, 5.2 \%, 6.9 \%$ and $19.7 \%$ without assuming jitter buffer and $0 \%, 5.2 \%, 5.3 \%$ and $18.3 \%$ with jitter buffer and increase call rate. No Packet loss rate in FTP traffic with increase call rate.

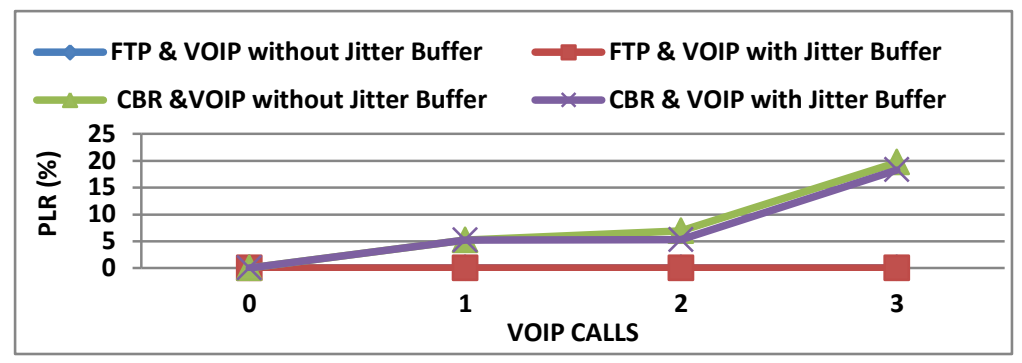

Figure 10. Packet Loss Rates Vs VolP Calls

Therefore only one channel is shared between voice and data traffic. Large number of packets of voice is send as compared with data traffic sender to destination. Data traffic sends only 1000 packets. Each voice sender sends more than 4000 packets $(4000 * 160$ bits = 640000 bits) of G711 audio data when call is established between senders to destination for 5 minute. With heavy load of data due to increased calls, throughput of data traffic decreases whereas average delay and average jitter of data traffic increases.

\section{Conclusion and Future Work}

This paper presents the performance evaluation of multi traffic over a key emerging technology (IEEE 802.11e) by using the network modeling and simulation tool QualNet 5.2. Simulations have been performed to investigate the effect of quality of service of multi traffic 
with changing parameters of multi traffics (CBR / FTP generic). It is observed that throughput of single CBR traffic is greater than a FTP generic. The throughput, average delay and average jitter of single CBR \& FTP traffic are $2.36 \mathrm{Mbps}, 0.038595 \mathrm{~s}$ and $0.001091 \mathrm{~s}$ respectively and $0.67989 \mathrm{Mbps}, 0.008889 \mathrm{~s}$ and $0.107346 \mathrm{~s}$ respectively. Increased call rate is affects the QoS of multi traffic (CBR \& FTP traffic). Throughput of traffic increase with decrease average delay and average jitter when payload size of CBR \& FTP traffic increased. Throughput of traffic decreases with increasing average delay, average jitter and packet loss rate when call rate increased. Dynamic jitter buffer used in VoIP codec has subtle improve quality of service of multi traffic and WLAN environments considered.

For future work, the performance can be considered and it can also be performance for HCCA with real time application. This technique can be further applied on mobiles nodes.

\section{References}

[1] IEEE Std. 802.11-1999: 'Part 11: Wireless LAN Medium Access Control (MAC) and Physical Layer (PHY) Specifications', (1999).

[2] N. M. Shafiul Kabir Chowdhury, Md. S. Hussain and F. Ahmed, "Finding the Performance Bottleneck of the IEEE802.11e EDCA Mechanism on Single Access Point (AP) Based WLAN Systems", ICECE 2010, (2010) December.

[3] T. Joshi, D. Ahuja, D. Singh and D. Agrawal, "SARA: Stochastic Automata Rate Adaptation for IEEE 802.11 Networks", IEEE Trans., (2008).

[4] B. Rauf, M. F. Amjad and K. Ahmed, "Performance Evaluation of IEEE 802.11 DCF in Comparison with IEEE 802.11e EDCA”, ICITST 2009, International Conference for (2009), pp. 1-6.

[5] A. Abbas, M. Hussain and T. Mubeen, "Medium Access and Flow Management in IEEE 802.11 and 802.11e WLANS", Advanced Computer Control, 2009. ICACC '09 on (2009), pp. 98-102.

[6] A. Abbas, T. Mubeen and S. Rizwan-ul-Hassan, "Capacity analysis for voice and queue management differences in IEEE 802.11 and 802.11e WLAN standards", Electrical Engineering, 2008, ICEE 2008, Second International Conference on (2008), pp. 1-5.

[7] M. Koprivica and M. Ilic, "Experimental Evaluation of IEEE 802.11e EDCA QoS Mechanism for Voice over WLAN", EUROCON - International Conference on Computer as a Tool (EUROCON), 2011 IEEE on (2011), pp. 1-4.

[8] S. Garg and M. Kappes, "An Experimental Study of Throughput for UDP and VoIP Traffic in IEEE 802.11b Networks", To appears in proceeding of the IEEE communication and Networking conference (WCNC), (2003), pp. 1748-1753.

[9] A. Syaheda, W. Marzuki, Y. Chai, H. Zen, L. Liang Wee and K. Lias, "Performances analysis of VoIP over 802.11b and 802.11e using different CODECs", Communications and Information Technologies (ISCIT), 2010 International Symposium on (2010), pp. 244-24.

[10] A. Khan, D.Smith, S. Hussein and H. Helgert, "Performance Analysis of VoIP codecs over multi-rate EDCA”, CCNC 2012 IEEE, (2012), pp. 110-115.

[11] "Qualnet Programmers Guide", scalable network technologies.

\section{Authors}

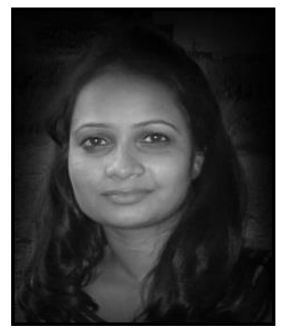

MEENAL, received her B.Tech degree in Electronics and Communication Engineering from Punjab Technical University, Jalandhar, India in 2009. She is presently pursuing MTECH from same university since 2010 and also working as lecturer in Faculty of Electronics and Communication Engineering, Pt. Jagat Ram Govt. Polytechnic College, Hoshiarpur, Punjab, India. The current focus of her research interests is on QoS of wireless local area networks(WLAN). 


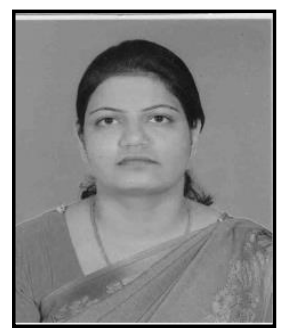

KIRAN AHUJA has done outstanding research in the fields of optical and wireless communication. She received her B.Tech \& M.Tech degree in Electronics and Communication Engineering from Punjab Technical University, Jalandhar, India and pursuing $\mathrm{Ph}$. D in Electronics \& Communication Engineering in wireless communication and networks field. She worked as faculty at DAVIET, Jalandhar, Punjab, India. 